\title{
Intake of individual saturated fatty acids and risk of coronary heart disease in US men and women: two prospective longitudinal cohort studies
}

\author{
Geng Zong,, Yanping Li, ${ }^{1}$ Anne J Wanders, ${ }^{2}$ Marjan Alssema, ${ }^{2}$ Peter L Zock, ${ }^{2}$ Walter C Willett, ${ }^{3}$ \\ Frank B Hu, ${ }^{3}$ Qi Sun ${ }^{4}$
}

Department of Nutrition, Harvard TH Chan School of Public Health, Boston, MA, USA ¿Unilever Research and Development, 3133AT,

Vlaardingen, Netherlands

${ }^{3}$ Departments of Nutrition and

Epidemiology, Harvard T H Chan

School of Public Health,

Channing Division of Network

Medicine, Department of

Medicine, Brigham and Women's

Hospital and Harvard Medical

School, Boston, MA, USA

${ }^{4}$ Department of Nutrition,

Harvard T H Chan School of

Public Health, Channing

Division of Network Medicine,

Department of Medicine,

Brigham and Women's Hospital

and Harvard Medical School,

665 Huntington Avenue,

Boston, MA 02115, USA

Correspondence to: Q Sun qisun@hsph.harvard.edu

Additional material is published online only. To view please visit the journal online.

Cite this as: $B M J$ 2016;355:15796 http://dx.doi.org/10.1136/bmj.i5796

Accepted: 21 October 2016

\section{ABSTRACT}

OBJECTIVES

To investigate the association between long term intake of individual saturated fatty acids (SFAs) and the risk of coronary heart disease, in two large cohort studies.

DESIGN

Prospective, longitudinal cohort study.

SETTING

Health professionals in the United States.

\section{PARTICIPANTS}

73147 women in the Nurses' Health Study (1984-2012) and 42635 men in the Health Professionals Follow-up Study (1986-2010), who were free of major chronic diseases at baseline.

\section{MAIN OUTCOME MEASURE}

Incidence of coronary heart disease $(\mathrm{n}=7035)$ was self-reported, and related deaths were identified by searching National Death Index or through report of next of kin or postal authority. Cases were confirmed by medical records review.

\section{RESULTS}

Mean intake of SFAs accounted for 9.0-11.3\% energy intake over time, and was mainly composed of lauric acid (12:0), myristic acid (14:0), palmitic acid (16:0), and stearic acid (18:0; 8.8-10.7\% energy). Intake of

\section{WHAT IS ALREADY KNOWN ON THIS TOPIC}

Saturated fat intake is a risk factor for coronary heart disease, and the replacement of saturated fat with unsaturated fat or whole grains has been associated with lower disease risk

Intervention studies have found that major saturated fatty acids in the diet, including lauric acid (12:0), myristic acid (14:0), palmitic acid (16:0), and stearic acid (18:0), had different effects on blood lipids

Little is known about association between intake of individual saturated fatty acids and risk of coronary heart disease in large cohort studies

\section{WHAT THIS STUDY ADDS}

Lauric acid, myristic acid, palmitic acid, and stearic acid are associated with an increased risk of coronary heart disease, after multivariate adjustment of covariates Risk of coronary heart disease is significantly lower when replacing the sum of these four major saturated fatty acids with polyunsaturated fat, whole grain carbohydrates, or plant proteins, with the lowest risk observed when palmitic acid, the most abundant saturated fatty acid, was replaced

Because intake of major saturated fatty acids are highly correlated, current dietary recommendations should focus on replacing total saturated fat with unsaturated fats or whole grain carbohydrate, as an effective approach towards preventing coronary heart disease

12:0, 14:0, 16:0 and 18:0 were highly correlated, with Spearman correlation coefficients between 0.38 and 0.93 (all $\mathrm{P}<0.001$ ). Comparing the highest to the lowest groups of individual SFA intakes, hazard ratios of coronary heart disease were 1.07 (95\% confidence interval 0.99 to $\left.1.15 ; P_{\text {trend }}=0.05\right)$ for $12: 0,1.13$ (1.05 to 1.22; $\left.P_{\text {trend }}<0.001\right)$ for $14: 0,1.18$ (1.09 to 1.27 ; $\left.P_{\text {trend }}<0.001\right)$ for $16: 0,1.18$ (1.09 to $\left.1.28 ; P_{\text {trend }}<0.001\right)$ for $18: 0$, and 1.18 (1.09 to 1.28; $\left.P_{\text {trend }}<0.001\right)$ for all four SFAs combined (12:0-18:0), after multivariate adjustment of lifestyle factors and total energy intake. Hazard ratios of coronary heart disease for isocaloric replacement of $1 \%$ energy from 12:0-18:0 were 0.92 (95\% confidence interval 0.89 to $0.96 ; \mathrm{P}<0.001)$ for polyunsaturated fat, 0.95 ( 0.90 to $1.01 ; P=0.08$ ) for monounsaturated fat, 0.94 (0.91 to 0.97; $P<0.001$ ) for whole grain carbohydrates, and 0.93 ( 0.89 to $0.97 ; P=0.001)$ for plant proteins. For individual SFAs, the lowest risk of coronary heart disease was observed when the most abundant SFA, 16:0, was replaced. Hazard ratios of coronary heart disease for replacing $1 \%$ energy from $16: 0$ were 0.88 ( $95 \%$ confidence interval 0.81 to 0.96 ; $\mathrm{P}=0.002$ ) for polyunsaturated fat, 0.92 ( 0.83 to 1.02 ; $\mathrm{P}=0.10)$ for monounsaturated fat, 0.90 (0.83 to 0.97; $\mathrm{P}=0.01)$ for whole grain carbohydrates, and $0.89(0.82$ to $0.97 ; \mathrm{P}=0.01$ ) for plant proteins.

\section{CONCLUSIONS}

Higher dietary intakes of major SFAs are associated with an increased risk of coronary heart disease. Owing to similar associations and high correlations among individual SFAs, dietary recommendations for the prevention of coronary heart disease should continue to focus on replacing total saturated fat with more healthy sources of energy.

\section{Introduction}

Prevailing dietary guidelines recommend keeping saturated fatty acid (SFA) intake below $10 \%$ of total energy for the prevention of cardiovascular disease, a leading cause of death worldwide. ${ }^{12}$ However, findings from recent systematic reviews and meta-analyses remain inconsistent on the association between SFA intake and coronary heart disease, largely owing to SFA being compared to different macronutrients in published studies. ${ }^{3-6}$ When SFA was replaced by polyunsaturated fat, lower risk of coronary heart disease has been observed in large scale prospective studies and intervention studies. ${ }^{56}$ But in practice, calories from SFA have mainly been replaced with low quality carbohydrate, ${ }^{7}$ which exert clear adverse effects on cardiometabolic disorders such as obesity and diabetes. ${ }^{8}$ 
Therefore, increased SFA intake did not appear to be associated with risk of coronary heart disease in many studies because the comparison nutrient was typically refined carbohydrates.

Intervention studies have consistently linked SFA intake with deteriorated blood lipid profile, with individual SFAs conferring heterogeneous effects, which might also explain the current controversies over SFAs. ${ }^{910}$ For example, a recently updated meta-analysis of clinical trials found that lauric acid (12:0), myristic acid (14:0), and palmitic acid (16:0) significantly raised levels of total cholesterol and low density lipoprotein (LDL) cholesterol when mixed carbohydrates in diet were replaced by these fatty acids. ${ }^{910}$ The effects of stearic acid (18:0), however, were largely neutral. ${ }^{910}$ Few observational studies have investigated the association between individual SFAs and risk of coronary heart disease. ${ }^{11-15}$ Our previous analysis of women in the Nurses' Health Study reported that intake of major SFAs (including 12:0, 14:0, 16:0, and 18:0) were associated with an elevated risk of coronary heart disease, whereas the sum of butyric acid (4:0), caproic acid (6:0), caprylic acid (8:0), and capric acid (10:0) was not. ${ }^{11}$

In the current study, we updated the analyses on associations between intake of individual SFAs and risk of coronary heart disease in the Nurses' Health Study with an extended follow-up of 18 years. We also included data from a cohort of men participating in the Health Professionals Follow-up Study. Most importantly, we estimated risk of coronary heart disease when individual SFAs were replaced by macronutrients that could help prevent coronary heart disease, ${ }^{716}$ including polyunsaturated fat, monounsaturated fat, whole grain carbohydrates, and plant proteins.

\section{Methods}

\section{Study population}

The Nurses' Health Study included 121700 female nurses aged 30-55 years in 1976, ${ }^{17}$ and the Health Professionals Follow-up Study included 51529 male health professionals aged 40-75 years in $1986 .{ }^{18}$ Information on medical history, lifestyle, potential risk factors, and disease diagnosis was collected at baseline through a self-administered questionnaire and updated every two years by use of similar questionnaires in both cohorts. The study protocol was approved by the institutional review boards of Brigham and Women's Hospital and the Harvard T H Chan School of Public Health. Return of self-administered questionnaires was considered informed consent.

The current analysis was conducted among 81757 women and 51529 men who completed a food frequency questionnaire at study baseline (years 1984 for the Nurses' Health Study and 1986 for the Health Professionals Follow-up Study). Participants were excluded if any of the following occurred:

- They reported physician diagnosed cancer, diabetes, or cardiovascular disease at study baseline $(n=7749$, Nurses' Health Study; n=7596, Health Professionals Follow-up Study).
- Their total energy intake was deemed implausible ( $<600$ or $>3500 \mathrm{kcal} /$ day, Nurses' Health Study; $<800$ or $>4200 \mathrm{kcal} /$ day, Health Professionals Follow-up Study; $1 \mathrm{kcal}=4.18 \mathrm{~kJ}$ ) or had missing individual SFA data $(n=98 ; n=147)$.

- They answered the baseline questionnaire only or had missing age at baseline $(n=763$, Nurses' Health Study; $n=1152$, Health Professionals Follow-up Study).

The final sample consisted of 73147 women and 42635 men with complete information.

\section{Ascertainment of diet}

In 1980, Nurses' Health Study participants completed a 61 item food frequency questionnaire on their usual intake of foods and beverages in the past year. In 1984 , 1986, and every four years thereafter until 2010, they were sent an expanded food frequency questionnaire to assess and update dietary information. The same questionnaire was sent to Health Professionals Follow-up Study participants every four years, from 1986 to 2010. The study baseline year was 1984 for the Nurses' Health Study, for more comprehensive estimates of individual SFA intake starting from this follow-up cycle. Overall, women completed and returned $6.9(86.3 \%)$ of the eight food frequency questionnaires received up to 2010, whereas men completed and returned 5.5 (78.6\%) of seven questionnaires received.

Participants were asked how often, on average, they had consumed specific foods in the past year, with nine responses ranging from "never" to "at least six times per day" based on a specified standard portion size. They were also asked about the types of fat, oil, and margarine used during cooking and at the table. Intakes of even-chain SFAs with carbon chain lengths between four and 18, total polyunsaturated fat, total monounsaturated fat, and total trans fat were calculated by multiplying the frequency of the consumption of each food item by its fatty acid composition, and then summing up values from all foods.

SFA composition of foods was based on the US Department of Agriculture and Harvard University food composition database, which is updated over time to reflect the nutrient profile of new food items and changes in processing. ${ }^{19}$ As detailed previously, we estimated whole grain carbohydrates on the basis of whole grain ingredients from a list of grain based foods. These foods include oatmeal; oatmeal, oat bran, or whole bran bread; brown rice; popcorn; whole wheat bread; whole wheat crackers; rye bread; oat based cold cereals; raw oat or wheat bran; and bran muffins. ${ }^{720}$

To better represent long term habitual intake and to minimize within-person variation, we calculated cumulative averages of food intake from all available dietary questionnaires throughout the follow-up period. ${ }^{21}$ We stopped updating diet information after participants reported a diagnosis of diabetes, stroke, or cancer, to minimize the possibility of reverse causation bias. Cumulative means of dietary variables before diagnosis of these diseases were then carried forward to represent 
diet for later follow-up. Missing values of individual SFAs were replaced with cumulative means of previous assessments. Fat intake estimates by food frequency questionnaires were validated against diet records over multiple weeks or $24 \mathrm{~h}$ dietary recall at baseline and during follow-up. $22-24$

The accuracy of individual SFA intake was evaluated in our latest validation study conducted in Nurses' Health Study between 2010 and 2012, and multivariate adjusted Spearman rank correlation coefficients were between 0.50 and 0.70 for total and individual SFA intakes. ${ }^{25}$ For example, correlations between estimates by food frequency questionnaires and seven day diet records were 0.69 (95\% confidence interval 0.62 to 0.73 ) for total SFAs, 0.70 (0.64 to 0.74 ) for 16:0, and 0.68 (0.61 to 0.73 ) for 18:0. For correlation coefficients between food frequency questionnaire estimates and those by repeat $24 \mathrm{~h}$ diet recalls, corresponding correlation coefficients were 0.71 ( 0.60 to 0.80 ), 0.74 (0.63 to 0.82 ), and 0.70 (0.60 to 0.79 ), respectively. ${ }^{25}$

Macronutrients were presented and analyzed as percentages of total energy intake, by dividing energy from the nutrients by total energy intake. Evaluating macronutrients as a percentage of energy is widely used in clinical settings and dietary guidelines. ${ }^{26}$ This approach helps control for confounding by total energy intake and are of more biological relevance, because effects of the same amount of nutrients could depend on body size, which is a major determinant of energy requirement. ${ }^{26}$

\section{Ascertainment of coronary heart disease}

In this study, total coronary heart disease included non-fatal myocardial infarction and fatal coronary heart disease. ${ }^{27}$ To ascertain non-fatal myocardial infarction, we first obtained permission of access to medical records from participants who reported having a physician diagnosed heart disease in follow-up questionnaires. Medical records were reviewed by study physicians who were blinded to exposure status, and telephone interviews were performed when medical records were not provided. Non-fatal myocardial infarction was confirmed using World Health Organization criteria of typical symptoms plus either elevated enzymes or diagnostic electrocardiography changes. ${ }^{28}$ We identified deaths by reports from next of kin and US postal authorities, or by searching the National Death Index. More than $98 \%$ of deaths can be identified by these approaches. ${ }^{27}$

Fatal coronary heart disease was confirmed by a review of hospital records or autopsy reports if coronary heart disease was listed as the underlying cause of death and if evidence of previous coronary heart disease was available from medical records. Probable fatal coronary heart disease was assigned if coronary heart disease was listed as the underlying cause of death on the death certificate but no medical records concerning the death were available and no prior reports of coronary heart disease was indicated. For this analysis, we included both confirmed and probable cases to maximize statistical power, and performed a sensitivity analysis by excluding probable fatal cases.

\section{Statistical analysis}

To describe the trend of individual SFA intake over time, we calculated aged adjusted intake of individual SFAs during follow-up without generating cumulative averages, carrying overof missing values, or stopping the updating of diets after chronic diseases were diagnosed. Because of their minor amounts and shared food sources, 4:0, 6:0, 8:0, and 10:0 were combined together as 4:0-10:0. Similarly, in addition to analyzing long chain SFAs individually, we calculated and used the groups 12:0-14:0 and 12:0-18:0.

Person years of follow-up were calculated from the return date of the baseline questionnaire to the date when participants were diagnosed with coronary heart disease, the date of death, or the end of follow-up, whichever came first. We estimated hazard ratios and $95 \%$ confidence intervals of incident coronary heart disease, according to individual SFA intake (divided into five groups based on quintiles), by using time dependent Cox proportional hazards regression model in each cohort with follow-up duration as the timescale. Analyses were conducted in the two cohorts separately; results were then pooled with a fixed effect model if the $P$ value for heterogeneity was greater than 0.05 .

Cox regression analysis was stratified jointly by age in months and calendar year to better control for confounding by age, calendar time, and any possible two way interactions between them. The multivariate model was adjusted for ethnicity, family history of myocardial infarction, body mass index, cigarette smoking, alcohol intake, physical activity, multivitamin use, menopausal status and postmenopausal hormone use (for women), current aspirin use, baseline hypertension, baseline hypercholesterolemia, and total energy intake. We did a test for linear trend by modeling median values of categories of individual SFAs as continuous variables.

Potential isocaloric substitution effect was estimated in a multivariate energy density model. This model further adjusted for energy from trans fat, polyunsaturated fat, monounsaturated fat, whole grain carbohydrates, non-whole grain carbohydrates, plant proteins, nonplant proteins, and the sum of SFAs except the one at issue. By leaving one specific SFA out of the model, regression coefficients of other macronutrients could be interpreted as estimated effects of isocalorically substituting one of these nutrients for that specific SFA while holding other macronutrients unchanged. In the substitution model, we adjusted for the sum of energy proportions from all these macronutrients as total energy intake.

We further estimated absolute risk reduction when mean individual SFA intake decreased from the highest to the lowest group, in isocaloric replacement by other nutrients. In this analysis, we first calculated the difference in mean SFA intake between the highest and lowest groups, and then derived the relative risk reduction of coronary heart disease corresponding to this change using hazard ratios in the substitution model above (estimated effects of $1 \%$ energy substitution). We multiplied this relative risk reduction with the incidence rate of coronary heart disease in the highest SFA 
intake group, in order to estimate the absolute risk reduction when specific SFA intake decreased from the highest to the lowest group with isocaloric replacement by another nutrient. ${ }^{2930}$ SFA intake and incidence rate were based on pooled study populations of men and women.

We tested proportional hazard assumption by including interaction terms between individual SFAs and follow-up duration in the model, and results did not suggest that the assumption was violated $(\mathrm{P}>0.05)$. Substitution analyses were stratified by age ( $<65$ years, $\geq 65$ years), body mass index $(<25, \geq 25)$, physical activity $(<18, \geq 18$ metabolic equivalent of task/week), and smoking status (current smoking or not). We performed three sensitivity analyses to examine the robustness of our findings. Firstly, we controlled for baseline body mass index instead of updated body mass index, because obesity is a potential mediator between SFAs and risk of coronary heart disease. Secondly, we adjusted for hypertension, hypercholesterolemia, and diabetes diagnosed during follow-up. Finally, we excluded probable fatal cases. Statistical analyses were performed by using SAS 9.4 (SAS Institute). All P values were two sided, with statistical significance defined as $\mathrm{P}<0.05$.

\section{Patient involvement}

No participants were involved in raising research questions or the outcome measures, nor were they involved in developing plans for recruitment, design, or implementation of the study. No participants were asked to advise on interpretation or writing up of the manuscript. Findings from the Nurses' Health Study and Health Professionals Follow-up Study have been posted on study websites and disseminated to participants as mailed newsletters annually (www.nurseshealthstudy.org and https://www.hsph.harvard.edu/hpfs/index.html).

\section{Results}

At baseline, participants with higher SFA intake were younger, were more likely to be white and non-smokers, had lower alcohol consumption, engaged in less physical activity, and had lower prevalence of hypercholesterolemia and hypertension than those with lower SFA intake (table 1). These participants were also less likely to take multivitamin supplements; had a higher body mass index; and had higher intakes of total energy, polyunsaturated fat, monounsaturated fat, trans fat, and proteins, and lower intakes of carbohydrates and plant proteins.

Proportions of energy intake from SFAs decreased from $11.3 \%$ in the Nurses' Health Study and $10.4 \%$ in the Health Professionals Follow-up Study at baseline to 9.1\% and $9.0 \%$ in 1998, respectively, and slightly increased again to $9.9 \%$ and $9.8 \%$ in 2010 , respectively (fig 1). Overall, 12:0, 14:0, 16:0, and 18:0 are the main SFAs in diet and account for 8.8-10.7\% energy on average. Throughout follow-up, mean proportion of energy from $16: 0$ was $5.7 \%$ for both women and men, followed by $18: 0$ (2.6\% for both sexes) and 14:0 (0.9\% for women and $0.8 \%$ for men). Lauric acid (12:0) and 4:0-10:0 con- tributed to $0.2 \%$ and $0.5 \%$ energy in men and women. Individual SFAs in the diet were highly correlated; Spearman correlation coefficients ranged from 0.38 between 4:0-10:0 and 18:0, to 0.93 between $16: 0$ and 18:0 (all $\mathrm{P}<0.001$; supplementary table 1 ).

Median follow-up duration was 25.8 (interquartile range 24.2-26.0, range 0.1-27.9) years for women and 21.2 (18.9-21.8, 0.1-23.7) years for men. During 2.72 million person years of follow-up, 7035 incident cases of coronary heart disease were identified (4348 with non-fatal disease, and 2687 with fatal disease). The sum intake of 4:0-10:0 was positively associated with risk of coronary heart disease in the age adjusted model $\left(\mathrm{P}_{\text {trend }}=0.009\right)$, which was attenuated after multivariate adjustment $\left(P_{\text {trend }}=0.30\right.$; table 2). Intakes of 12:0, 14:0, 16:0, 18:0, and the sum of 12:0-18:0 were all associated with a higher risk of coronary heart disease in age and multivariate adjusted models (all $\mathrm{P}_{\text {trend }}<0.05$ ). Pooled hazard ratios of coronary heart disease comparing the highest to the lowest intake groups were 1.07 (95\% confidence interval 0.99 to 1.15 ; $\mathrm{P}_{\text {trend }}=0.05$ ) for 12:0, 1.13 (1.05 to 1.22; $\left.\mathrm{P}_{\text {trend }}<0.001\right)$ for 14:0, 1.18 (1.09 to 1.27; $\left.\mathrm{P}_{\text {trend }}<0.001\right)$ for $16: 0,1.18$ (1.09 to $\left.1.28 ; \mathrm{P}_{\text {trend }}<0.001\right)$ for $18: 0$, and 1.18 (1.09 to 1.28 ; $\left.\mathrm{P}_{\text {trend }}<0.001\right)$ for 12:0-18:0 in the multivariate adjusted model.

We further estimated risk of coronary heart disease when 12:0, 14:0, 16:0, and 18:0 were isocalorically replaced by polyunsaturated fat, monounsaturated fat, whole grain carbohydrates, or plant proteins. As shown in fig 2, isocalorically replacing $1 \%$ energy from 16:0 with total polyunsaturated fat, whole grain carbohydrates, or plant proteins was significantly associated with a lower risk of coronary heart disease. Pooled hazard ratios of coronary heart disease were 0.88 (95\% confidence interval 0.81 to $0.96 ; \mathrm{P}=0.002$ ), 0.90 ( 0.83 to $0.97 ; \mathrm{P}=0.01$ ), and 0.89 (0.82 to 0.97 ; $\mathrm{P}=0.01$, respectively.

Corresponding to the reduction of 16:0 intake from the highest intake group to the lowest, estimated absolute risk reduction was 93.4 cases of coronary heart disease per 100000 person years when 16:0 was replaced by polyunsaturated fat, 79.5 cases per 100000 person years by whole grain carbohydrates, and 86.5 cases per 100000 person years by plant proteins. For each $1 \%$ energy substitution for 16:0, the estimated absolute risk reductions were 34.4 cases per 100000 person years for polyunsaturated fat, 28.7 cases per 100000 person years for whole grain carbohydrates, and 31.5 cases per 100000 person years for plant proteins.

There was also a non-significant trend towards a reduction in risk of coronary heart disease (hazard ratio 0.92 (95\% confidence interval 0.84 to $1.01 ; \mathrm{P}=0.07$ ) when $1 \%$ energy from 18:0 was isocalorically replaced by polyunsaturated fat, which corresponded to 38.4 cases of coronary heart disease per 100000 person years, an absolute risk reduction of 23.5 cases per 100000 person years for $1 \%$ energy replacement.

After 12:0, 14:0, 16:0, and 18:0 were pooled together, isocaloric replacement of 12:0-18:0 by more healthy nutrients was associated with a reduced risk of coronary heart disease. Hazard ratios were 0.92 (95\% confidence 


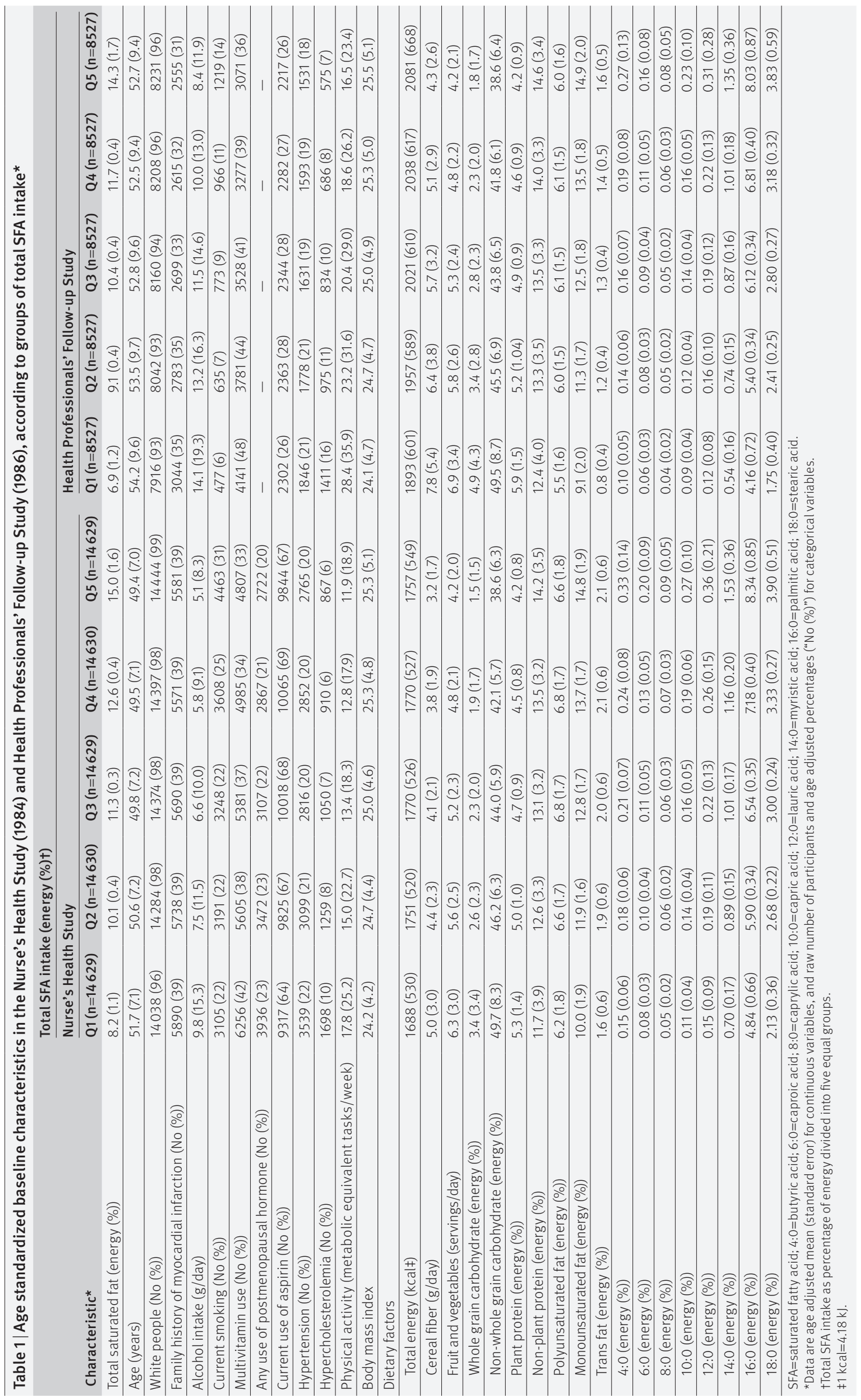



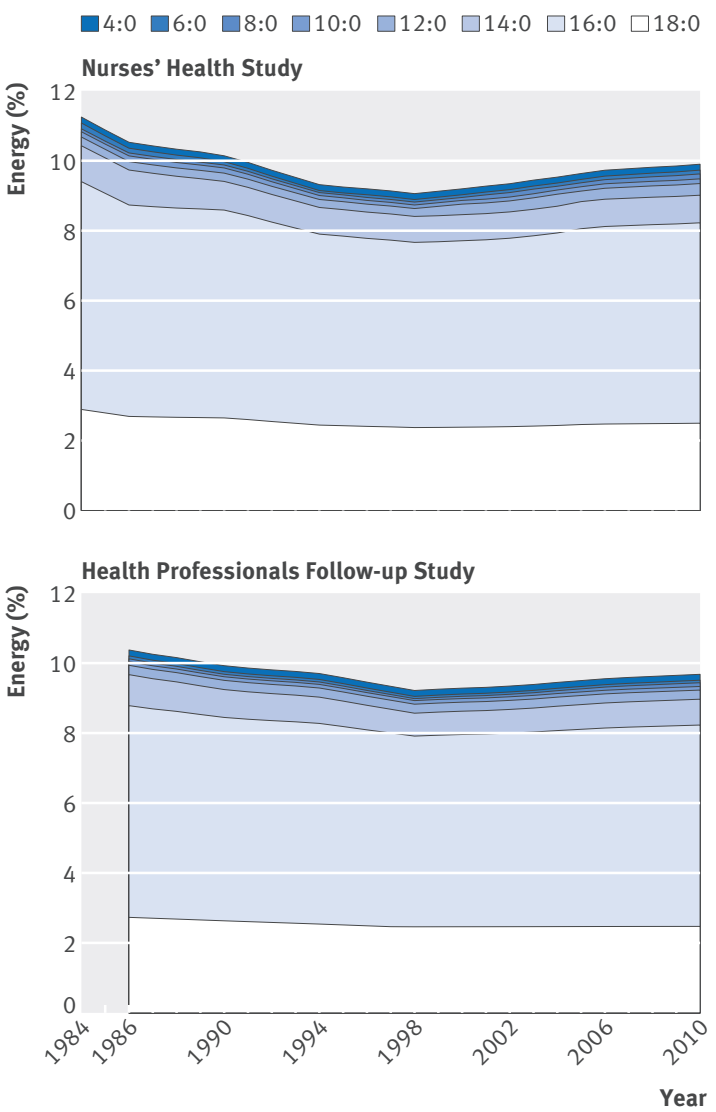

Fig 1 | Aged adjusted intake (energy (\%)) of individual saturated fatty acids over time in the Nurses' Health Study (1984-2010) and Health Professionals Follow-up Study (1986-2010). 4:0=butyric acid; 6:0=caproic acid; 8:0=caprylic acid; 10:0=capric acid; $12: 0=$ lauric acid; 14:0=myristic acid; $16: 0=$ palmitic acid; 18:0=stearic acid

interval 0.89 to 0.96 ; $\mathrm{P}<0.001)$ for polyunsaturated fat, 0.95 ( 0.90 to 1.01; $\mathrm{P}=0.08$ ) for monounsaturated fat, 0.94 (0.91 to 0.97; $\mathrm{P}<0.001$ ) for whole grain carbohydrates, and 0.93 ( 0.89 to $0.97 ; \mathrm{P}=0.001$ ) for plant proteins. When mean 12:0-18:0 intake decreased from the highest intake group to the lowest, absolute risk reduction was estimated to be 106.2 cases of coronary heart disease per 100000 person years when replaced by polyunsaturated fat, 70.9 cases per 100000 person years by monounsaturated fat, 83.2 cases per 100000 person years by whole grain carbohydrates, and 94.9 cases per 100000 person years by plant proteins. For each $1 \%$ energy substitution, these risk reductions were 23.4 cases per 100000 person years for polyunsaturated fat, 14.6 cases per 100000 person years for monounsaturated fat, 17.5 cases per 100000 person years for whole grain carbohydrates, and 20.4 cases per 100000 person years for plant proteins. There was no significant heterogeneity in results between the two cohorts.

Substitution analysis was stratified by age, body mass index, physical activity, and smoking status (supplementary table 2), and all $\mathrm{P}$ values for interaction were greater than 0.05. Associations between SFA intake and risk of coronary heart disease were similar when baseline body mass index was adjusted as a covariate (supplementary table 3), but were slightly attenuated when incident hypertension, hypercholesterolemia, and diabetes diagnosed during follow-up were further adjusted (supplementary table 4). Exclusion of probable fatal cases of coronary heart disease $(n=790)$ did not materially change the main findings (supplementary table 5).

\section{Discussion \\ Principal findings}

In two large prospective cohorts of US men and women, dietary intakes of major individual SFAs-including lauric acid (12:0), myristic acid (14:0), palmitic acid (16:0), and stearic acid (18:0) - were positively associated with risk of coronary heart disease during 24-28 years of follow-up. Replacement of 1\% daily energy intake from the combined group of 12:0-18:0 by equivalent energy from polyunsaturated fat, whole grain carbohydrates, or plant proteins was associated with a 6-8\% reduced risk of coronary heart disease. The same replacement of 16:0 was associated with $10-12 \%$ reduction in risk.

\section{Strengths and weaknesses in relation to other studies}

The present analysis, to our knowledge, provides the largest observational study so far to examine the association between intake of individual SFAs and risk of coronary heart disease. An early ecological study showed that populations with higher intakes of 12:0, 14:0, 16:0, and 18:0 had a higher mortality from coronary heart disease, but it is not clear whether these associations were independent of other lifestyle and dietary factors. ${ }^{13}$ In a case-control study of 933 Costa Rican people, dietary intake of 12:0, 14:0,16:0, and 18:0 was positively associated with prevalent acute myocardial infarction, even after adjustment for unsaturated fat, trans fat, and proteins. ${ }^{12}$ Two recent studies from the Netherlands reported largely diverging findings. In the European Prospective Investigation into Cancer and Nutrition study, intakes of 4:0-10:0 and 12:0 were inversely associated with ischemic heart disease risk, ${ }^{14}$ but no associations were found for 14:0, 16:0, and 18:0. However, in the Rotterdam study, only 16:0 intake was associated with higher risk of coronary heart disease. ${ }^{15}$ Based on a 14-year follow-up in the Nurses' Health Study, our previous analysis showed that risk of coronary heart disease was 29\% higher when intake of 12:0-18:0 increased by $5 \%$ energy. ${ }^{11}$ In the current study, which had extended follow-up in the Nurses' Health Study and additional data from the Health Professionals Follow-up Study, our analyses replicated the finding that substitution of healthy macronutrients for 12:0-18:0 was associated with a reduced risk of coronary heart disease.

In a meta-analysis of prospective studies, de Souza and colleagues did not find significant associations between total saturated fat intake and coronary heart disease, but noticed large heterogeneity among estimates from different studies. ${ }^{3}$ The most plausible reason for such heterogeneity is the inconsistent adjustment of covariates (especially macronutrients) in the individual studies. ${ }^{3}$ As a result, the associations 


\section{Table 2 | Association between intake of individual SFAs and risk of coronary heart disease in the Nurses' Health Study (1984-2010) and Health} Professionals Follow-up Study (1986-2010)

\section{SFA intake (energy (\%))*}

Q1 $\mathbf{Q 2}$

4:0-10:0

Nurses' Health Study

\begin{tabular}{l} 
Median SFA intake (energy (\%)) \\
\hline CHD/person year (No) \\
\hline Age adjusted model† \\
\hline Multivariate model \\
\hline Health Professionals Follow-up Study \\
\hline Median SFA intake (energy (\%))
\end{tabular}

Median SFA intake (energy (\%))

$\mathrm{CHD} /$ person year ( $\mathrm{No})$

Age adjusted model

Multivariate model

Pooled analysis§

Age adjusted model

Multivariate model

12:0

Nurses' Health Study

Median SFA intake (energy (\%))

CHD/person year (No)

Age adjusted model

Multivariate model

Health Professionals Follow-up Study

Median SFA intake (energy (\%))

$\mathrm{CHD} /$ person year (No)

Age adjusted model

Multivariate model

Pooled analysis

Age adjusted model

14:0

0.30

$587 / 370588$

0.41

$1 \quad 1.02(0.91$ to 1.15$)$

$1.02(0.91$ to 1.15$)$

Q3

Q4

Q5

$P_{\text {trend }}$

$0.21 \quad 0.32$

$811 / 173021$

$766 / 173450$

0.99 (0.89 to 1.09$)$

1.00 (0.90 to 1.10$)$

1.00 (0.93 to 1.08$)$

1.03 (0.95 to 1.11)

0.12

$593 / 370058$

0.16

556/370 205

0.99 (0.88 to 1.11$)$

1.01 (0.90 to 1.14)

0.50

$560 / 370466$

0.61

0.99 (0.88 to 1.11)

$583 / 370160$

0.82

1.01 (0.90 to 1.14)

1.04 (0.93 to 1.17$)$

1.06 (0.95 to 1.20$)$

$7 / 368728$

1.13 (1.01 to 1.27)

1.08 (0.96 to 1.21$)$

0.02

0.41

$791 / 173160$

0.52

1.00 (0.91 to 1.11$)$

$819 / 173069$

1.00 (0.91 to 1.11)

1.03 (0.93 to 1.14$)$

1.02 (0.93 to 1.13 )

0.73

$902 / 172314$

1.06 (0.96 to 1.16$)$

1.01 (0.92 to 1.12)

0.14

$1.00(0.93$ to 1.08$)$

1.03 (0.96 to 1.11)

1.01 (0.93 to 1.09$)$

1.04 (0.96 to 1.12$)$

1.09 (1.01 to 1.17) 0.009

1.04 (0.96 to 1.12$) \quad 0.30$

(2)

0.10

$844 / 172454$

0.14

$789 / 173116$

1.00 (0.90 to 1.10$)$

0.99 (0.90 to 1.10$)$

$565 / 370484$

0.24

$572 / 370234$

(0.92 to 1.16$)$

1.05 (0.94 to 1.18$)$

1.03 (0.92 to 1.16$)$

0.37

$660 / 369659$

1.05 (0.93 to 1.18)

0.22

$794 / 173261$

1.02 (0.93 to 1.13)

$781 / 173342$

1.02 (0.92 to 1.12)

1.01 (0.91 to 1.11)

1.00 (0.90 to 1.10$)$

1.23 (1.10 to 1.38$)$

1.11 (0.99 to 1.24)

$<0.001$

0.07

$1 \quad 0.99(0.92$ to 1.07$)$

1.03 (0.95 to 1.11)

1.02 (0.95 to 1.10)

0.34

$881 / 172840$

$1.10(1.00$ to 1.21$)$

1.04 (0.95 to 1.15$)$

0.33

$1.00(0.93$ to 1.08$)$

1.03 (0.95 to 1.11)

1.01 (0.94 to 1.09$)$

$1.15(1.07$ to 1.24$)$

$<0.001$

1.07 (0.99 to 1.15$)$

0.05

Nurses' Health Study

\begin{tabular}{|c|c|c|c|c|c|c|}
\hline Median SFA intake (energy (\%)) & 0.63 & 0.79 & 0.91 & 1.05 & 1.33 & - \\
\hline CHD/person year (No) & $596 / 370997$ & $558 / 370854$ & $534 / 370521$ & $605 / 369940$ & $653 / 368328$ & - \\
\hline Age adjusted model & 1 & $1.02(0.91$ to 1.15$)$ & $1.03(0.91$ to 1.15$)$ & 1.17 (1.05 to 1.31$)$ & 1.28 (1.14 to 1.43$)$ & $<0.001$ \\
\hline Multivariate model & 1 & 1.01 (0.89 to 1.13$)$ & 0.99 (0.88 to 1.12$)$ & $1.08(0.97$ to 1.22$)$ & $1.10(0.98$ to 1.23$)$ & 0.04 \\
\hline \multicolumn{7}{|l|}{ Health Professionals Follow-up Study } \\
\hline Median SFA intake (energy (\%)) & 0.50 & 0.68 & 0.80 & 0.95 & 1.22 & - \\
\hline CHD/person year (No) & $775 / 173398$ & 754/173399 & $809 / 173246$ & $812 / 172832$ & $939 / 172138$ & - \\
\hline Age adjusted model & 1 & 1.06 (0.95 to 1.17) & 1.17 (1.06 to 1.29$)$ & $1.17(1.06$ to 1.30$)$ & 1.29 (1.17 to 1.42$)$ & $<0.001$ \\
\hline Multivariate model & 1 & $1.03(0.93$ to 1.14$)$ & $1.12(1.01$ to 1.24$)$ & 1.09 (0.98 to 1.21) & 1.15 (1.04 to 1.27$)$ & 0.004 \\
\hline \multicolumn{7}{|l|}{ Pooled analysis } \\
\hline Age adjusted model & 1 & 1.04 (0.96 to 1.12) & 1.11 (1.02 to 1.19) & 1.17 (1.09 to 1.27$)$ & 1.28 (1.19 to 1.38$)$ & $<0.001$ \\
\hline Multivariate model & 1 & 1.02 (0.94 to 1.10$)$ & 1.06 (0.98 to 1.15$)$ & 1.09 (1.01 to 1.17) & 1.13 (1.05 to 1.22$)$ & $<0.001$ \\
\hline
\end{tabular}

16:0

Nurses' Health Study

\begin{tabular}{l} 
Median SFA intake (energy (\%)) \\
\hline CHD/person year (No) \\
Age adjusted model \\
Multivariate model \\
Health Professionals Follow-up Study \\
Median SFA intake (energy (\%))
\end{tabular}

Median SFA intake (energy (\%))

CHD/person year (No)

Age adjusted model

Multivariate model

$68-5.49$

$586 / 370403$

$574 / 370902$

$\begin{array}{ll}1 & 1.10(0.98 \text { to } 1.24) \\ 1 & 1.07(0.95 \text { to } 1.20)\end{array}$

$569 / 370506$

1.18 (1.05 to 1.32$)$

6.61

1.08 (0.96 to 1.21$)$

1.29 (1.15 to 1.45$)$

7.55

4.22

5.21

$772 / 173340 \quad 767 / 173368 \quad 786 / 173172$

$1.08(0.98$ to 1.20$)$

$1.16(1.05$ to 1.28$)$

6.52

1.10 (0.99 to 1.22$)$

$840 / 172897$

1.16 (1.05 to 1.28$)$

$626 / 368685$

$1.45(1.29$ to 1.62$)$

$1.12(1.00$ to 1.27$) \quad<0.001$

Pooled analysis

Age adjusted model

Multivariate model

$1.05(0.95$ to 1.17$)$

$1.16(1.08$ to 1.26$)$

1.28 (1.18 to 1.38$)$

1.15 (1.06 to 1.24$)$

7.52

18:0

$1.09(1.01$ to 1.18$)$
$1.06(0.98$ to 1.15$)$

1.09 (1.01 to 1.18$)$

$924 / 172237$

Nurses' Health Study

Median SFA intake (energy (\%))

CHD/person year (No)

2.02

2.46

$553 / 370679$

$557 / 370728$

2.76

$547 / 370516$

3.07

$634 / 370053$

3.56

$655 / 368663$

(Continued) 


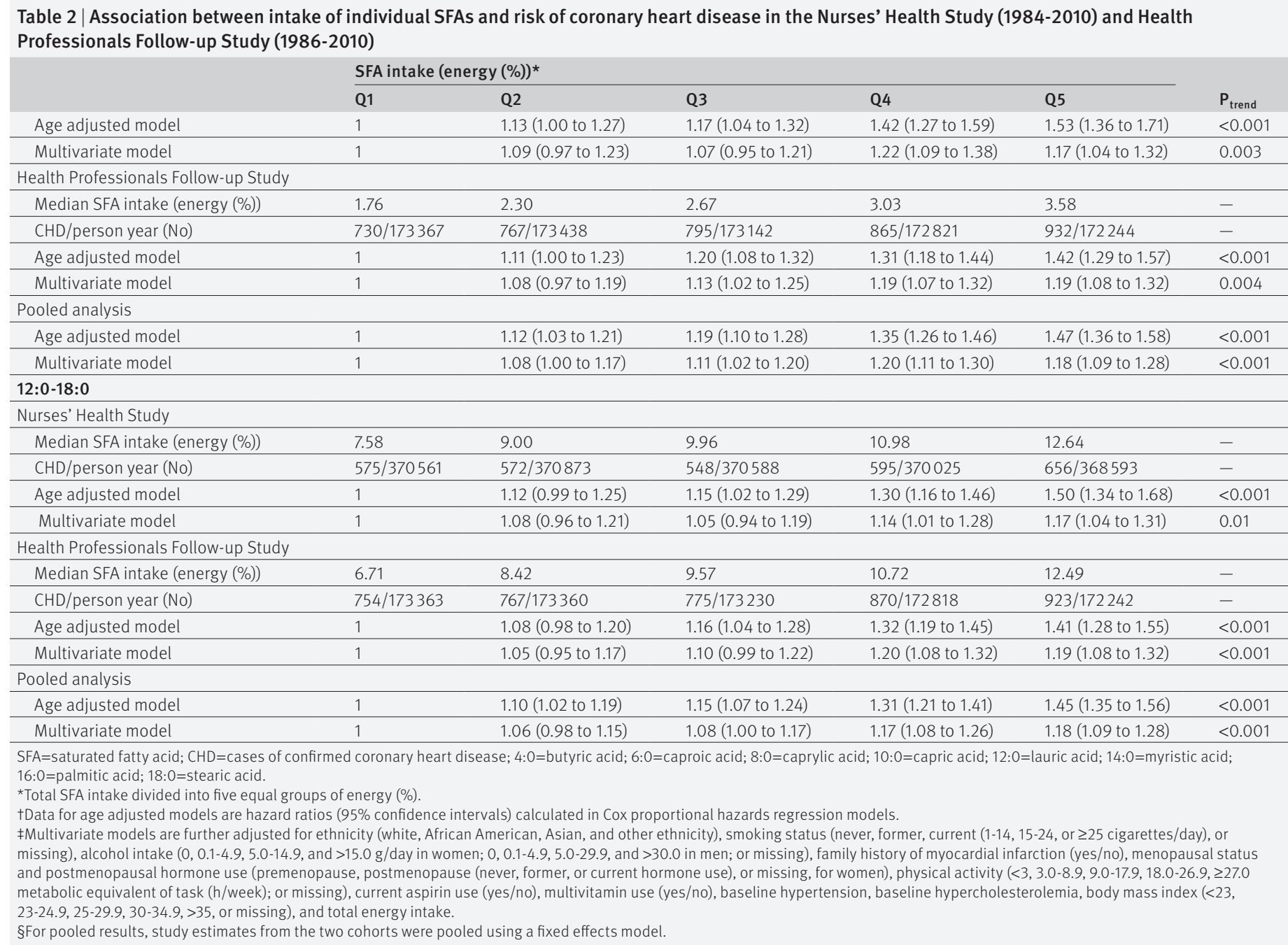

could reflect the effects of substituting SFA for various other macronutrients (depending on the statistical modeling in individual studies), with mixed health effects. Most importantly, without a specified replacement, the comparison is largely with refined starch and sugar because these are the dominant sources of calories in the US diet.

Our previous analysis in the Nurses' Health Study and the Health Professionals Follow-up Study found a consistently lower risk of coronary heart disease when total saturated fat was replaced by polyunsaturated fat, monounsaturated fat, or whole grain carbohydrates, whereas replacing SFAs with refined carbohydrate was not associated with a lower risk. ${ }^{7}$ A previous pooling analysis of 11 prospective cohort studies that specified the substituting macronutrient also observed that incidence of coronary heart disease and mortality was 26\% and 13\% lower, respectively, when 5\% energy from saturated fat was replaced by polyunsaturated fat. ${ }^{5}$ Such findings are in line with a meta-analysis of eight large clinical trials reporting that replacement of 5\% energy as saturated fat with polyunsaturated fat led to a $19 \%$ reduction in coronary heart disease risk during a median follow-up duration of 4.5 years. ${ }^{6}$

\section{Possible explanations and implications}

Mensink recently updated the meta-analysis of clinical trials on individual SFA intakes and blood lipids, and found that replacing 1\% energy from carbohydrates with 16:0 was associated with $0.04 \mathrm{mmol} / \mathrm{L}$ increase in LDL cholesterol $(\mathrm{P}<0.001)$, but no significant change was observed for 18:0 $(-0.003 \mathrm{mmol} / \mathrm{L}, \mathrm{P}=0.61) .{ }^{10}$ This difference could be one reason for the stronger associations between 16:0 and coronary heart disease in the present substitution model. Alternatively, given that relative risks for individual SFAs were not largely different in our study (especially those for 16:0 and 18:0), stronger association for 16:0 might simply reflect much higher intake of 16:0 relative to other SFAs, thus enabling more robust risk estimates. We also found that the risk of coronary heart disease was lower when 18:0 was replaced by polyunsaturated fat among women, but not in men or in the pooled data, nor was the between-study heterogeneity significant. In intervention studies, both 12:0 and 14:0 increase LDL cholesterol when replacing energy from carbohydrate. ${ }^{10}$ However, replacement of 12:0-14:0 with other beneficial macronutrients was not associated with a reduced risk of coronary heart disease in the current study, which 


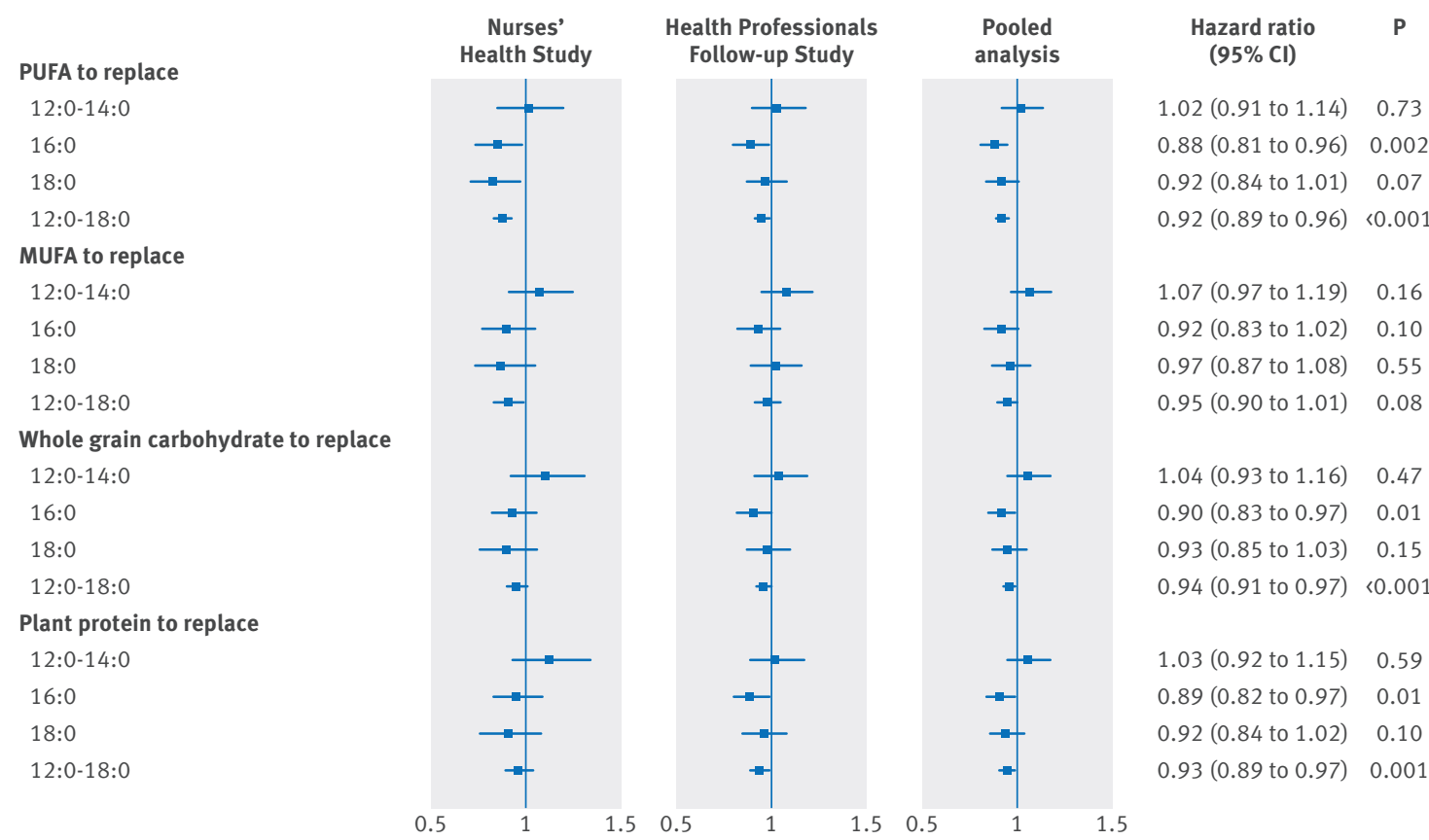

Fig 2 | Multivariate adjusted hazard ratios of coronary heart disease after substitution of $1 \%$ of energy from individual SFAs by alternative nutrients, based on the Nurses' Health Study (1984-2012) and Health Professionals Follow-up Study (1986-2010). Dots=hazard ratio point estimates; horizontal bars=95\% confidence intervals; SFA=saturated fatty acid; 12:0=lauric acid; 14:0=myristic acid; 16:0=palmitic acid; 18:0=stearic acid; PUFA=polyunsaturated fatty acids; MUFA=monounsaturated fatty acids. Hazard ratios were adjusted for age, ethnicity (white, African American, Asian, and other ethnicity), smoking status (never, former, current (1-14, 15-24, or $\geq 25$ cigarettes/day), or missing), alcohol intake (0, 0.1-4.9, 5.0-14.9, and $>15.0 \mathrm{~g} /$ day in women; 0, 0.1-4.9, 5.0-29.9, and $>30.0 \mathrm{~g} /$ day in men; or missing), family history of myocardial infarction (yes/no), menopausal status and postmenopausal hormone use (premenopause, postmenopause (never, former, or current hormone use), or missing, for women), physical activity ( $<3,3.0-8.9,9.0-17.9,18.0-26.9, \geq 27.0$ metabolic equivalent of task (h/week); or missing), current aspirin use (yes/no), multivitamin use (yes/no), baseline hypertension, baseline hypercholesterolemia, body mass index $(<23,23-24.9,25-29.9,30-34.9,>35$, or missing), total energy intake as sum of energy from all included macronutrients, energy from trans fat, energy from carbohydrate of non-whole grain sources, and energy from non-plant sources. For PUFA (polyunsaturated fatty acids) replacement, hazard ratios were further adjusted for MUFA (monounsaturated fatty acids), whole grain carbohydrates, plant proteins, and the sum of other SFAs. For MUFA replacement, hazard ratios were further adjusted for PUFA, whole grain carbohydrates, plant proteins, and the sum of other SFAs. For whole grain carbohydrate replacement, hazard ratios were further adjusted for PUFA, MUFA, plant proteins, and the sum of other SFAs. For plant protein replacement, hazard ratios were further adjusted for PUFA, MUFA, whole grain carbohydrates, and the sum of other SFAs. Study estimates from two cohorts were pooled using a fixed effects model

could be explained by a much lower abundance of 12:0 and 14:0 in the diet. ${ }^{10}$ In addition, we cannot exclude the possibility that the non-significant findings regarding 12:0 and 14:0 were due to collinearity among SFAs.

Collectively, our study supports current dietary guidelines that focus on reducing total saturated fat intake with unsaturated fats, which for example, can be achieved by replacing animal fats (eg, butter, lard) with vegetable oils high in unsaturated fat (eg, olive oil, canola oil). Some snacks (eg, crackers, chips, and popcorns), bakery products (eg, cakes, cookies, and pastry), and table spread (eg, margarine) are also important dietary sources of SFAs, ${ }^{31}$ because the production and storage of these foods require fat that is stable at room or cooking temperature. ${ }^{32}$ These foods are produced not only by food industry but also by small scale workshops (such as bakeries), restaurants, and home cooking, and could vary substantially in terms of individual SFA contents depending on the specific fats and oils used. . $^{334}$

Palm oil, the most commonly produced edible oil in the world, contains over $40 \%$ fat as $16: 0$, whereas coconut oil and palm kernel oil are high in 12:0 (over $45 \%$ fat). ${ }^{33}$ Therefore, it is feasible to modulate the fatty acid profiles of foods by selecting oils or fats used in preparation. ${ }^{33}$ Several lines of evidence have suggested increased use of palm oil in food production, especially in developing countries, ${ }^{35}$ and higher 16:0 contents in snacks and bakery products over the past years. ${ }^{3637}$ Such trends might lead to adverse consequences, ${ }^{9}$ and calls for integrated and effective solutions that involve food producers, consumers, and policymakers. According to an economic-epidemiological model, taxation on palm oil in India could prevent 363000 cardiovascular deaths over 10 years. ${ }^{35}$ Meanwhile, whether fats and oils high in 18:0 could be less harmful substitutes for trans fat and other SFAs remains to be confirmed. ${ }^{38} 39$

\section{Strengths and weaknesses}

The strengths of our study include large sample sizes; long follow-up duration; repeated measurements of diet, lifestyle, and health status; and the use of cumulative average intakes of nutrients to reflect long 
term diet. ${ }^{21}$ Several limitations should be discussed as well. Firstly, although we adjusted for a multitude of covariates, including demographic characteristics, lifestyle, medical history, and dietary factors, potential unmeasured and residual confounding cannot be excluded. Secondly, our study populations exclusively consisted of health professionals with a relatively homogeneous ethnic background and socioeconomic status. Although this renders the study results less susceptible to confounding by ethnicity and socioeconomic factors, such homogeneity could limit the generalizability of findings to other ethnic groups or populations with different socioeconomic profiles. Thirdly, dietary data derived from food frequency questionnaires are measured, inevitably, with errors. Because of the prospective design, these errors are most likely to bias true associations towards the null. Lastly, it is difficult to disentangle associations of individual SFAs that are highly correlated with each other in this observational study setting. Potential differential effects of individual SFAs on cardiovascular risk warrant further investigation in other cohort studies.

\section{Conclusion}

In summary, we found dietary replacement of 12:0-18:0 with more healthy macronutrients-such as polyunsaturated fat and whole grain carbohydrates-was associated with a lower risk of coronary heart disease. Particularly, the replacement of 16:0 with these beneficial macronutrients was associated with the lowest risk. Owing to high correlations among individual SFAs in diet, these findings support the current dietary recommendations that focus on replacement of total saturated fat as an effective approach to preventing cardiovascular disease. The public health and clinical significance of modulating the content of individual SFAs in specific foods should be further evaluated.

Contributors: QS, WCW, and FBH obtained funding from the National Institutes of Health. QS and FBH designed the study. QS, WCW, and FBH were involved in data collection. GZ, YL, and QS provided statistical expertise. GZ analysed the data and wrote the first draft of the manuscript. All authors contributed to the interpretation of the results and critical revision of the manuscript for important intellectual content and approved the final version of the manuscript. GZ and QS are the guarantors of this investigation.

Funding: This study was funded by research grants UM1 CA186107, R01 HL034594, R01 HL35464, R01 HL60712, and UM1 CA167552 from the National Institutes of Health. QS was supported by a career development award (ROO-HL098459) from the National Heart, Lung, and Blood Institute. The sponsors have no role in: the study design; the collection, analysis, or interpretation of data; the writing of the report; or in the decision to submit the article for publication.

Competing interests: All authors have completed the ICMJE uniform disclosure form at www.icmje.org/coi_disclosure.pdf and declare: support from the National Institutes of Health for the submitted work; GZ is supported by a postdoctoral fellowship funded by Unilever R\&D, Vlaardingen, Netherlands; AJW, MA, and PLZ are employees of Unilever R\&D (Unilever is a producer of food consumer products); FBH has received research support from California Walnut Commission and Metagenics; no other relationships or activities that could appear to have influenced the submitted work.

Ethical approval: The study protocol was approved by the institutional review boards of the Brigham and Women's Hospital and the Harvard TH Chan School of Public Health. The completion of the self-administered questionnaire was considered to imply informed consent.
Data sharing: No additional data available.

The lead author confirms that the manuscript is an honest, accurate and transparent account of the study being reported; that no important aspects of the study have been omitted; and that any discrepancies from the study as planned (and, if relevant, registered) have been explained.

This is an Open Access article distributed in accordance with the Creative Commons Attribution Non Commercial (CC BY-NC 3.0) license, which permits others to distribute, remix, adapt, build upon this work non-commercially, and license their derivative works on different terms, provided the original work is properly cited and the use is non-commercial. See: http://creativecommons.org/licenses/ by-nc/3.0/.

Mozaffarian D, Benjamin EJ, Go AS, et al. American Heart Association Statistics Committee and Stroke Statistics Subcommittee. Heart disease and stroke statistics-2015 update: a report from the American Heart Association. Circulation 2015;131:e29-322. doi:10.1161/CIR.0000000000000152.

2 Astrup A, Dyerberg J, Elwood P, et al. The role of reducing intakes of saturated fat in the prevention of cardiovascular disease: where does the evidence stand in 2010? Am J Clin Nutr 2011;93:684-8. doi:10.3945/ajcn.110.004622.

3 de Souza RJ, Mente A, Maroleanu A, et al. Intake of saturated and trans unsaturated fatty acids and risk of all cause mortality, cardiovascular disease, and type 2 diabetes: systematic review and meta-analysis of observational studies. BMJ 2015;351:h3978. doi:10.1136/bmj.h3978.

4 Siri-Tarino PW, Sun Q, Hu FB, Krauss RM. Meta-analysis of prospective cohort studies evaluating the association of saturated fat with cardiovascular disease. Am J Clin Nutr 2010;91:535-46. doi:10.3945/ ajcn.2009.27725

5 Jakobsen MU, O'Reilly EJ, Heitmann BL, et al. Major types of dietary fat and risk of coronary heart disease: a pooled analysis of 11 cohort studies. Am J Clin Nutr 2009;89:1425-32. doi:10.3945/ ajcn.2008.27124.

6 Mozaffarian D, Micha R, Wallace S. Effects on coronary heart disease of increasing polyunsaturated fat in place of saturated fat: a systematic review and meta-analysis of randomized controlled trials. PLoS Med 2010;7:e1000252. doi:10.1371/ journal.pmed.1000252.

$7 \quad$ Li Y, Hruby A, Bernstein AM, et al. Saturated fats compared with unsaturated fats and sources of carbohydrates in relation to risk of coronary heart disease: a prospective cohort study. I Am Coll Cardiol 2015;66:1538-48. doi:10.1016/j.jacc.2015.07.055.

8 Hu FB. Are refined carbohydrates worse than saturated fat? Am J Clin Nutr 2010;91:1541-2. doi:10.3945/ajcn.2010.29622.

9 Mensink RP, Zock PL, Kester AD, Katan MB. Effects of dietary fatty acids and carbohydrates on the ratio of serum total to HDL cholesterol and on serum lipids and apolipoproteins: a meta-analysis of 60 controlled trials. Am / Clin Nutr 2003·77:1146-55.

10 Mensink RP. Effects of saturated fatty acids on serum lipids and lipoproteins: a systematic review and regression analysis. World Health Organization, 2016.

11 Hu FB, Stampfer MJ, Manson JE, et al. Dietary saturated fats and their food sources in relation to the risk of coronary heart disease in women. Am J Clin Nutr 1999;70:1001-8.

12 Kabagambe EK, Baylin A, Siles X, Campos H. Individual saturated fatty acids and nonfatal acute myocardial infarction in Costa Rica. Eur J Clin Nutr 2003;57:1447-57. doi:10.1038/sj.ejcn.1601709.

13 Kromhout D, Menotti A, Bloemberg B et al. Dietary saturated and trans fatty acids and cholesterol and 25-year mortality from coronary heart disease: the Seven Countries Study. Prev Med 1995;24:308-15. doi:10.1006/pmed.1995.1049.

14 Praagman J, Beulens JW. Alssema M, et al. The association between dietary saturated fatty acids and ischemic heart disease depends on the type and source of fatty acid in the European Prospective Investigation into Cancer and Nutrition-Netherlands cohort. Am / Clin Nutr 2016;103:356-65. doi:10.3945/ajcn.115.122671.

15 Praagman J, de Jonge EA, Kiefte-de Jong JC, et al. Dietary saturated fatty acids and coronary heart disease risk in a Dutch middle-aged and elderly population. Arterioscler Thromb Vasc Biol 2016;36:2011. 8. doi:10.1161/ATVBAHA.116.307578.

16 Song M, Fung TT, Hu FB, et al. Association of animal and plant protein intake with all-cause and cause-specific mortality. JAMA Intern Med 2016;176:1453-63. doi:10.1001/jamainternmed.2016.4182.

17 Hu FB, Manson JE, Stampfer MJ, et al. Diet, lifestyle, and the risk of type 2 diabetes mellitus in women. N Engl / Med 2001:345:790-7. doi:10.1056/NEJMoa010492

18 van Dam RM, Willett WC, Rimm EB, Stampfer MJ, Hu FB. Dietary fat and meat intake in relation to risk of type 2 diabetes in men. Diabetes Care 2002:25:417-24. doi:10.2337/diacare.25.3.417.

19 Farvid MS, Cho E, Chen WY, Eliassen AH, Willett WC. Dietary protein sources in early adulthood and breast cancer incidence: prospective cohort study. BMJ 2014;348:g3437. doi:10.1136/bmj.g3437. 
20 Jensen MK, Koh-Banerjee P, Hu FB, et al. Intakes of whole grains, bran, and germ and the risk of coronary heart disease in men. Am J Clin Nutr 2004;80:1492-9.

21 Hu FB, Stampfer MJ, Rimm E, et al. Dietary fat and coronary heart disease: a comparison of approaches for adjusting for total energy intake and modeling repeated dietary measurements. Am J Epidemiol 1999;149:531-40. doi:10.1093/oxfordjournals.aje.a009849.

22 Salvini S, Hunter DJ, Sampson L, et al. Food-based validation of a dietary questionnaire: the effects of week-to-week variation in food consumption. Int JEpidemiol 1989;18:858-67. doi:10.1093/ije/18.4.858.

23 Feskanich D, Rimm EB, Giovannucci EL, et al. Reproducibility and validity of food intake measurements from a semiquantitative food frequency questionnaire. / Am Diet Assoc 1993;93:790-6. doi:10.1016/0002-8223(93)91754-E.

24 Garland M, Sacks FM, Colditz GA, et al. The relation between dietary intake and adipose tissue composition of selected fatty acids in US women Am / Clin Nutr 1998:67:25-30.

25 Yuan C, Spiegelman D, Rimm EB, et al. Nutrient validation utilizing self-reported dietary assessment methods in women's lifestyle validation study. Am / Epidemiol 2016 [forthcoming].

26 Willett W. Implications of total energy intake for epidemiologic analyses. Nutritional epidemiology. 3rd ed. Oxford University Press, 2013.

27 Chiuve SE, Sampson L, Willett WC. The association between a nutritional quality index and risk of chronic disease. Am J Prev Med 2011;40:505-13. doi:10.1016/j.amepre.2010.11.022

28 Rose GA. Cardiovascular survey methods. 2nd ed. World Health Organization, 1982

29 Eliassen AH, Colditz GA, Rosner B, Willett WC, Hankinson SE. Adult weight change and risk of postmenopausal breast cancer. JAMA 2006;296:193-201. doi:10.1001/jama.296.2.193.

30 Hamajima N, Hirose K, Tajima K, et al. Collaborative Group on Hormonal Factors in Breast Cancer. Alcohol, tobacco and breast cancer-collaborative reanalysis of individual data from 53 epidemiological studies, including 58,515 women with breast cancer and 95,067 women without the disease. Br J Cancer 2002;87:1234-45. doi:10.1038/sj.bjc.6600596.
31 O’Neil CE, Keast DR, Fulgoni VL, Nicklas TA. Food sources of energy and nutrients among adults in the US: NHANES 2003-2006. Nutrients 2012:4:2097-120. doi:10.3390/nu4122097.

32 Eckel RH, Borra S, Lichtenstein AH, Yin-Piazza SY. Trans Fat Conference Planning Group. Understanding the complexity of trans fatty acid reduction in the American diet: American Heart Association Trans Fat Conference 2006: report of the Trans Fat Conference Planning Group. Circulation 2007;115:2231-46. doi:10.1161/ CIRCULATIONAHA.106.181947.

33 Dubois V, Breton S, Linder M, et al. Fatty acid profiles of 80 vegetable oils with regard to their nutritional potential. Eur J Lipid Sci Technol 2007;109:710-32. doi:10.1002/ejlt.200700040.

34 Tarrago-Trani MT, Phillips KM, Lemar LE, Holden IM. New and existing oils and fats used in products with reduced trans-fatty acid content. J Am Diet Assoc 2006;106:867-80. doi:10.1016/j. jada.2006.03.010.

35 Basu S, Babiarz KS, Ebrahim S, Vellakkal S, Stuckler D, GoldhaberFiebert JD. Palm oil taxes and cardiovascular disease mortality in India: economic-epidemiologic model. BMJ 2013;347:f6048 doi:10.1136/bmi.f6048.

36 Van Camp D, Hooker NH, Lin CT. Changes in fat contents of US snack foods in response to mandatory trans fat labelling. Public Health Nutr 2012;15:1130-7. doi:10.1017/S1368980012000079.

37 Trattner S, Becker W, Wretling S, Öhrvik V, Mattisson I. Fatty acid composition of Swedish bakery products, with emphasis on trans-fatty acids. Food Chem 2015;175:423-30. doi:10.1016/j. foodchem.2014.11.145.

38 Hunter JE, Zhang J, Kris-Etherton PM. Cardiovascular disease risk of dietary stearic acid compared with trans, other saturated, and unsaturated fatty acids: a systematic review. Am J Clin Nutr 2010;91:46-63. doi:10.3945/ajcn.2009.27661.

39 Willett WC. Dietary fats and coronary heart disease. J Intern Med 2012;272:13-24. doi:10.1111/j.1365-2796.2012.02553.x.

\section{Appendix: Supplementary tables}

